\title{
Next-generation sequencing of tissue and circulating tumor DNA: Resistance mechanisms to EGFR targeted therapy in a cohort of patients with advanced non-small cell lung cancer
}

\author{
Yujun Zhang \\ Shanghai Chest Hospital, Shanghai Jiao Tong University \\ Liwen Xiong \\ Shanghai Chest Hospital, Shanghai Jiao Tong University \\ Fangfang Xie \\ Shanghai Chest Hospital, Shanghai Jiao Tong University \\ Xiaoxuan Zheng \\ Shanghai Chest Hospital, Shanghai Jiao Tong University \\ Ying Li \\ Shanghai Chest Hospital, Shanghai Jiao Tong University \\ Lei Zhu \\ Shanghai Chest Hospital, Shanghai Jiao Tong University \\ Jiayuan Sun ( $\nabla$ xkyyjysun@163.com ) \\ Shanghai Jiao Tong University Affiliated Chest Hospital
}

Research

Keywords: pidermal growth factor receptor-tyrosine kinase inhibitor (EGFR-TKI), tissue biopsy, non-small cell lung cancer (NSCLC), genetic alterations, clinical outcomes

Posted Date: May 29th, 2020

DOI: https://doi.org/10.21203/rs.3.rs-30958/v1

License: (c) (7) This work is licensed under a Creative Commons Attribution 4.0 International License. Read Full License 


\section{Abstract \\ Background}

Epidermal growth factor receptor-tyrosine kinase inhibitor (EGFR-TKI) has been considered as an effective treatment in epidermal growth factor receptormutant (EGFR-mutant) advanced non-small cell lung cancer (NSCLC). However, most patients develop acquired resistance eventually. Here, we compared and analyzed the genetic alterations between tissue assay and circulating tumor DNA (ctDNA), and further explored the resistance mechanisms after EGFR-TKI treatment.

\section{Methods}

Amplification refractory mutation system-polymerase chain reaction (ARMS-PCR), Cobas ${ }^{\circledR}$ ARMS-PCR and next-generation sequencing (NGS) were performed on tissue samples after pathological diagnosis. Digital droplet PCR (ddPCR) and NGS were performed on plasma samples. The association between genetic alterations and clinical outcomes was analyzed retrospectively.

\section{Results}

Thirty-seven patients were included. The success rate of re-biopsy was $91.89 \%$ (34/37). The total detection rate of EGFR T790M was $62.16 \%$ (23/37) and the consistency between tissue and ctDNA was $78.26 \%$ (18/23). Thirty-four patients were analyzed retrospectively. Twenty-four patients harbored concomitant mutations. Moreover, tissue re-biopsy at resistance showed 21 patients $(21 / 34,61.76 \%)$ had concomitant T790M mutation, 4 with MET amplification and 4 with PIK3CA mutation. Patients with T790M mutation $(p=0.010 \& p=0.017)$ or third-generation EGFR-TKI treatment $(p<0.0001 \& p=0.073)$ showed better progression-free survival (PFS) and overall survival (OS). Interestingly, concomitant genetic alterations were significantly associated with a worse prognosis for patients with T790M mutation receiving third-generation EGFR-TKIs $(p=0.037)$.

\section{Conclusions}

Multi-platforms are feasible and highly consistent for re-biopsy after EGFR-TKI resistance. Concomitant genetic alterations may be associated with a poor prognosis for patients with T790M mutation after third-generation EGFR-TKIs.

\section{Trial Registration:}

The clinical trial registration was carried out on ClinicalTrials.gov. (NCT03309462), registered on September 1 st 2017.

https://register.clinicaltrials.gov/prs/app/action/SelectProtocol?sid=S0007G1B\&selectaction=Edit\&uid=U0001WUU\&ts=2\&cx=frpicv

\section{Background}

Lung cancer is the leading cause of cancerrelated mortality worldwide [1]. Among them, non-small cell lung cancer (NSCLC) accounts for about $85 \%$ lung cancer [2]. Epithelial growth factor receptor (EGFR) is the most common driver gene in NSCLC, occurring in an estimated $50 \%$ of adenocarcinoma cases in Asia [3]. Exon 19 deletion (19del) and exon 21 p.L858R (21L858R) mutation account for about 90\% of all EGFR activating mutations and are the most relevant predictors of response to EGFR tyrosine kinase inhibitor (EGFR-TKI) [4]

However, almost all patients eventually develop acquired drug resistance after EGFR-TKI treatment. And the occurrence of EGFR mutation p.T790M in exon 20 represents the most frequent mechanism of the acquired resistance [5]. The third-generation EGFR-TKI is an irreversible selective TKI, which specifically targets EGFR T790M and EGFR activating mutations, and has been proven effective in patients with EGFR T790M-positive NSCLC following acquired resistance to prior EGFR-TKIs $[6,7]$. This makes re-biopsy widely accepted in clinical practice [8]. Through the re-biopsy, it is possible to effectively understand the cause of drug resistance and provide a basis for follow-up treatment.

Unfortunately, resistance to third-generation EGFR-TKIs also inevitably occurs $[9,10]$. In addition, the detection rate of mutations has increased significantly with the development of next-generation sequencing (NGS), while the association of concomitant genetic alterations with the treatment response is still uncertain [11, 12]. Therefore, it is important to explore the status of re-biopsy to identify resistance mechanisms early in patients with targeted drugs.

\section{Methods}

\section{Patient selection}

Patients with advanced NSCLC, who were evaluated as progressive disease (PD) after the first-generation and the second-generation EGFR-TKI treatment from September 2017 to January 2019 with initial diagnosis of EGFR 19del or EGFR 21L858R mutation, were enrolled. The inclusion criteria included a.18-80 years old; b. patients with initial diagnosis of advanced NSCLC and molecular pathology confirmed the presence of EGFR-sensitive mutations; c. clinically accepted first and second-generation EGFR-TKI treatment; $d$. according to the criteria of solid tumor evaluation criteria (response evaluation criteria in solid tumors, RECIST), clinicians believe that re-biopsy is necessary to guide the treatment of the patient; e. patient performance status (PS) rated as score $\leq 2$ according to the Eastern Cooperative Oncology Group (ECOG). The exclusion criteria were as follows: a. the patient received blood transfusion therapy within 1 month; $b$. 
patients with autoimmune diseases, including but not limited to systemic lupus erythematosus, rheumatoid arthritis, Sjogren's syndrome, etc.; c. patients with serious diseases were not suitable for biopsy; $d$. patients refused to participate in clinical trials; e. the investigator believed that the patient had other conditions that were not suitable for this clinical trial. The study was approved by the Ethics Committee of Shanghai Chest Hospital, and the ethical approval number was KS1703. All patients were fully informed and signed informed consent. The clinical trial registration was carried out on ClinicalTrials.gov. (NCT03309462).

\section{Sample preparation}

Liquid biopsy specimens were collected via standard venipuncture techniques into two tubes. And circulating tumor DNA (ctDNA) was extracted from the plasma fraction of EDTA blood samples within two hours of collection as recommended (QIAamp Circulating Nucleic Acid Kit; Qiagen, Germantown, MD). Tissue samples were obtained from small biopsies with multiple techniques, including but not limited to transbronchial biopsy (TBB), transbronchial lung biopsy (TBLB), transbronchial needle aspiration (TBNA), transthoracic needle aspiration (TTNA), and percutaneous lymph node needle biopsy. Then the obtained tissue samples were fixed and embedded. After that, the formalin-fixed, paraffin-embedded (FFPE) specimens underwent histological review by hematoxylin eosin staining before subjecting to nucleic acid extraction. DNA was purified with the use of a QIAamp DNA FFPE Kit (Qiagen, Valencia, CA), and the quantity of DNA was verified with the use of Qubit 3.0 with a dsDNA HS Assay Kit (Life Technologies).

\section{Gene testing methods}

ARMS-PCR for detecting EGFR mutations was performed via EGFR 21 Mutations Detection Kit (Amoy Diagnostics, Xiamen, China). DNA was extracted from 10 to 15 unstained FFPE sections, each 5 m thickness, using QIAamp DNA FFPE tissue kit (Qiagen, Valencia, CA, USA) according to the manufacturer's instructions. The concentration of DNA was measured by SMA4000 spectrophotometer (Merinton, Beijing, China). Cobas ${ }^{\circledR}$ ARMS-PCR was performed with 10-15 slides of $5 \mu \mathrm{m}$ paraffin sections subjected to DNA extraction using a Cobas ${ }^{\circledR}$ DNA sample preparation kit (Roche Diagnostics, Indianapolis, USA), and DNA concentration and purity were examined using a Nanodrop UV-Vis spectrophotometer. DNA integrity was examined by agarose gel electrophoresis. Genetic testing was performed using the human EGFR gene kit Cobas ${ }^{\circledR}$ EGFR Mutation Test v2 (Roche Diagnostics, Indianapolis, USA) for EGFR 18-21 exons mutations. For NGS, the library was constructed based on OncoAim ${ }^{\text {TM }}$ cancer 223 gene panel (Singlera Genomics, Inc., Shanghai, China) with a total of 1300 exon regions, 456 hotspots, 21 intron regions, and 1 gene promoter. DNA shearing was performed using Covaris M220, followed by end repair, phosphorylation and adaptor ligation. Fragments of size 200-400 bp were selected using Agencourt AMPure beads (Beckman Coulter, Brea, CA, USA) followed by hybridization with capture probes baits, hybrid selection with magnetic beads and PCR amplification. A bio-analyzer high-sensitivity DNA assay was performed to assess the quality and size of the fragments. Fifty ng of DNA was used for library construction. Twelve PCR cycles were used for library amplification. The indexed samples were sequenced on Hiseq500 sequencer (IIlumina, Inc., San Diego, CA, USA) with paired reads (read length 150 bp). The sequencing data in the FASTQ format were mapped to the human genome (hg19) using BWA aligner 0.7.10. Local alignment optimization, variant calling and annotation were performed using GATK 3.2, MuTect, and VarScan, respectively. DNA translocation analysis was performed using both Tophat2 and Factera 1.4.3. Gene-level copy number variation was assessed using a t statistic after normalizing reads depth at each region by total reads number and region size, and correcting GCbias using a LOESS algorithm.

\section{Follow-up}

Clinical follow-up assessments including physical examinations, imaging, and routine laboratory tests were performed every 4 weeks. Tumor response was assessed according to the RECIST (version) 1.1 by experienced investigators and categorized as complete response (CR), partial response (PR), stable disease (SD) or PD. Progression-free survival (PFS) was defined as the duration between the initiation of EGFR-TKIs and progression of disease or death for any cause, whichever occurred first. Similarly, overall survival (OS) was calculated from the date of starting EGFR-TKIs to the date of death for any cause or last follow-up (censored patient). Patients who were still alive were censored on their date of last follow-up per chart review. The cut-off date for analysis was April $25,2019$.

\section{Statistical analysis}

Frequency, percentage, average \pm SD, median (range) were presented as appropriate. Survival was estimated with Kaplan-Meier methodology and compared with the use of log-rank test between different groups. P values were calculated using Fisher's exact test or Pearson test for categorical variables or continuous variables, respectively. Wilcoxon test was used for comparing continuous variables to binary variables. Statistical analysis was performed using SPSS v25 (IBM Corporation, NY, USA). A $P$ value less than 0.05 was considered statistically significant.

\section{Results}

\section{Patients' characteristics}

In this study, 39 patients were enrolled and 34 patients were included in the retrospective analysis. The enrollment process is shown in Fig. 1. Two patients were excluded, due to one 19del false positive and one T790M mutation at baseline. Thirty-seven patients were successfully tested for plasma with a success rate of $100 \%$ (37/37), while three patients failed to perform tissue re-biopsy due to few tumor cells by pathological examination. The characteristics of initial and secondary biopsy of enrolled patients were summarized in Tables 1 and 2 . Thirty-four patients' clinical characteristics for retrospective analysis were shown in Table 3. Pathology revealed only one adenosquamous cell carcinoma, the others were adenocarcinoma (97.06\%, 33/34). And the common EGFR activating alterations were largely represented (67.65\%, 23/34, 19del; 32.35\%, 11/34, 21L858R). During first-line treatment, median PFS resulted 13 months. After progression, 70.59\% (24/34) of patients received third-generation EGFR-TKI treatment, and median PFS resulted 6 months. The first patient started thirdgeneration EGFR-TKI treatment on October 24, 2017 and the last one assumed the first dose on August 17, 2018. The data cut-off for this analysis was April $25,2019$. 


\section{Characteristics}

Gender, n (\%)

Male

Female

Age, median (range), years

Smoking history, n (\%)

Yes

No

Pathology, n (\%)

Adenocarcinoma

adenosquamous carcinoma

Molecular pathology, n (\%)

EGFR 19de

EGFR 21L858R

Treatment, $\mathrm{n}(\%)$

First-generation EGFR-TKI

Chemotherapy + first-generation EGFR-TKI

$\mathrm{PFS}^{*}$, median (range), months

Sites, n (\%)

Lung lesions

Intrathoracic metastatic lymph nodes

Extrathoracic metastatic lymph nodes

Pleural effusion

Methods of initial biopsy, $\mathrm{n}(\%)$

TBB

EBUS-TBNA

TBLB

TTNA

Percutaneous needle aspiration of lymph nodes

Surgery

Pleural effusion

*PFS was determined from the starting date of the first-generation EGFR-TKI treatment to the date of disease progression.

Abbreviations: EBUS-TBNA, endobronchial ultrasound transbronchial needle aspiration; EGFR, epidermal growth factor receptor; EGFR-TKI, epidermal growth factor receptor- tyrosine kinase inhibitor; PFS, progression-free survival; TBB, transbronchial biopsy; TBLB, transbronchial lung biopsy; TTNA,

transthoracic needle aspiration

\section{Number}

$21(53.85 \%)$

$18(46.15 \%)$

$61(38-79)$

$12(30.77 \%)$

$27(69.23 \%)$

$38(97.44 \%)$

$1(2.56 \%)$

$26(66.67 \%)$

$13(33.33 \%)$

$36(92.31 \%)$

$3(7.69 \%)$

$13.0(1-100)$

$26(66.67 \%)$

3 (7.69\%)

$6(15.38 \%)$

4 (10.26\%)

10 (25.64\%)

3 (7.69\%)

1 (2.56\%)

$10(25.64 \%)$

$6(15.38 \%)$

$5(12.82 \%)$

4 (10.26\%) 


\section{Characteristics}

Pathology, n (\%)

Adenocarcinoma

Squamous cell lung cancer

Small cell lung cancer

Inadequate tumor cells

Clinical staging, $\mathrm{n}(\%)$

IIIA

IIIB

IV

Metastasis, $\mathrm{n}$

Bone metastasis

Brain metastasis

- Pleural metastasis

Other distant metastases

Treatment, $\mathrm{n}(\%)$

Third-generation EGFR-TKI

Radio/chemotherapy

$\mathrm{PFS}^{\star}$, median (range), months

OS, median (range), months

Sites, $\mathrm{n}(\%)$

Lung lesions

Intrathoracic metastatic lymph nodes

Extrathoracic metastatic lymph nodes

Methods of re-biopsy, $\mathrm{n}$ (\%)

TBB

EBUS-TBNA

TBLB

TTNA

Percutaneous needle aspiration of lymph nodes

\section{Number}

$30(81.08 \%)$

$3(8.11 \%)$

$1(2.7 \%)$

$3(8.11 \%)$

$3(8.11 \%)$

$2(5.41 \%)$

$32(86.49 \%)$

$11(29.73 \%)$

$16(43.24 \%)$

$8(21.62 \%)$

$18(48.65 \%)$

$27(72.97 \%)$

$10(27.03 \%)$

$6(0-23)$

$13(0-23)$

$23(62.16 \%)$

$8(21.62 \%)$

$6(16.22 \%)$

$9(24.32 \%)$

$8(21.62 \%)$

$6(16.22 \%)$

$8(21.62 \%)$

$6(16.22 \%)$

*PFS was determined from the starting date of the third-generation EGFR-TKI treatment and/or other treatments to the date of disease progression, or the last follow-up time for those who have not reached disease progression.

Abbreviations: EBUS-TBNA, endobronchial ultrasound transbronchial needle aspiration; EGFR-TKI, epidermal growth factor receptor- tyrosine kinase inhibitor; OS, overall survival; PFS, progression-free survival; TBB, transbronchial biopsy; TBLB, transbronchial lung biopsy; TTNA, transthoracic needle aspiration. 
Table 3

Patients' clinical characteristics for retrospective analysis

\section{Characteristic}

Age, mean $\pm S D$, years

Sex

Male

Female

Smoking history

Never smoker

Smoker

Clinical stage

[

T stage

T1

T2

T3

T4

Lymph node metastasis

NO

N2

N3

Distant metastasis

No

Yes

Metastatic sites

Brain

Bone

Liver

Adrenal

Pericardium

ECOG PS

0

1

Baseline pathological classification

Adenocarcinoma

Adenosquamous carcinoma

Re-biopsy pathological classification

Adenocarcinoma

Squamous carcinoma

Small cell lung cancer

EGFR mutation

19del
NO. (\%)

$58.5 \pm 9.31$

$17(50 \%)$

$17(50 \%)$

$27(79.41 \%)$

$7(20.59 \%)$

$5(14.71 \%)$

$29(85.29 \%)$

$1(2.94 \%)$

$22(64.71 \%)$

$2(5.88 \%)$

$9(26.47 \%)$

$1(2.94 \%)$

$16(47.06 \%)$

$17(50 \%)$

$5(14.71 \%)$

$29(85.29 \%)$

$10(29.41 \%)$

$17(50 \%)$

$2(5.88 \%)$

$4(11.76 \%)$

$2(5.88 \%)$

$12(35.29 \%)$

$22(64.71 \%)$

$33(97.06 \%)$

$1(2.94 \%)$

$30(88.24 \%)$

$3(8.82 \%)$

$1(2.94 \%)$

$23(67.65 \%)$

Abbreviations: ECOG PS: Eastern Cooperative Oncology Group performance status; EGFR, epidermal growth factor receptor; EGFR-TKI, epidermal growth factor receptor- tyrosine kinase inhibitor 


\begin{tabular}{|ll|}
\hline Characteristic & NO. (\%) \\
\hline L858R & $11(32.35 \%)$ \\
\hline First line EGFR-TKI & $8(23.53 \%)$ \\
\hline gefitinib & $3(8.82 \%)$ \\
\hline erlotinib & $21(61.76 \%)$ \\
\hline icotinib & $2(5.88 \%)$ \\
\hline others & \\
\hline Post-TKI treatment & $24(70.59 \%)$ \\
\hline Third-generation EGFR-TKI & $10(29.41 \%)$ \\
\hline Radio/chemotherapy & \\
\hline $\begin{array}{l}\text { Abbreviations: ECOG PS: Eastern Cooperative Oncology Group performance status; EGFR, epidermal growth factor receptor; EGFR-TKI, epidermal growth } \\
\text { factor receptor-tyrosine kinase inhibitor }\end{array}$ \\
\hline
\end{tabular}

\section{Genetic alterations in ctDNA and tissue of enrolled patients}

Of the 37 patients who had plasma sent for ctDNA NGS, 34 (91.89\%) also had tissue sent for solid tumor NGS. Out of 34 patients with samples sent for both ctDNA and tissue NGS, 34 had shared at least 1 alteration identified by both tissue NGS and ctDNA analysis. In Fig. 2A, the most frequent alterations detected by ctDNA NGS are displayed. The three most frequent ctDNA alterations involved the following genes: TP53 (67.57\%, 25/37), followed by KRAS (8.11\%, 3/37) and amplification of c-Met (5.41\%, 2/37) (Fig. 2B). The most frequent alterations detected by tissue NGS involved the following genes: TP53 (52.94\%, 18/34), amplification of c-Met $(11.76 \%, 4 / 34)$ and PIK3CA (11.76\%, 4/34) (Fig. 2C\&D). In total, 83.78\% (31/37) of patients harbored concomitant mutations, 70.27\% $(26 / 37)$ by ctDNA and $70.59 \%$ (24/34) by tissue NGS. Patients with a history of smoking $(85.71 \%$ [6/7] vs $66.67 \%$ [18/27]) was found in tissue with a higher incidence of concomitant mutations, but not in ctDNA (37.5\% [3/8] vs $79.31 \%$ [23/29]).

A total of 23 cases of EGFR T790M mutation in plasmas and tissues were detected after first-line EGFR-TKI treatment. The total positive rate was $62.16 \%$ (23/37). The positive rate of tissue samples to detect EGFR T790M mutation was $64.71 \%(22 / 34)$, and the positive rate of plasma samples to detect EGFR T790M mutation was $51.35 \%$ (19/37). One of the patients had a positive mutation in plasma, while the tissue was negative. This patient was treated with Osimertinib in the follow-up treatment. The best efficacy was evaluated as PR, and PFS was 7.0 months. Therefore, we combined the efficacy and test results to consider the tissue test results as false negative, suggesting that tissue and ctDNA assay provided complementary results. The remaining 18 patients with positive blood tests were consistent with those having positive tissue tests (Fig. 3A). Therefore, the consistency rate between tissue and plasma for EGFR T790M mutation was $78.26 \%$ (18/23). In addition, EGFR T790M mutation in plasma samples were tested and compared with ddPCR and NGS as illustrated by the Venn diagrams. Tissues were detected and compared by ARMS-PCR, Cobas ${ }^{\circledR}$ ARMS-PCR and NGS (Fig. 3B\&C). The consistency rate in plasma and tissue for different platforms were $84.21 \%(16 / 19)$ and $78.26 \%(18 / 23)$, respectively.

\section{Treatment outcome}

Of the 34 patients in the retrospective analysis, 24 received third-generation EGFR-TKI treatment and 10 received others after progression with first-line EGFRTKI treatment. Twenty-four $(70.59 \%, 24 / 34)$ of patients exhibited prominent tumor shrinkage during treatment. Among them, 22 received third-generation EGFR-TKI treatment, revealing the strength of third-generation EGFR-TKIs and radio/chemotherapy for patients with resistance to first-line treatment (Fig. 4). Thirteen partial responses were observed and all occurred in T790M-positive patients (contained one who was detected only by ctDNA assay and one was not detected by the tissue NGS but was detected on other platforms), the objective response rate (ORR) was $54.17 \%$ as shown in Table 4 ( $p=0.003$ ). Comparing with third-generation EGFR-TKI treatment, radio/chemotherapy(others) significantly showed shorter $\mathrm{PFS}(\mathrm{p}<0.001$, median survival, 4.0 months, ratio, $0.25[95 \% \mathrm{Cl}, 0.19-0.85$ months] vs 10.0 months, ratio, 4[95\%Cl, 1.17-5.34]; HR, 4.49[95\%Cl, 1.36-14.76])and 0S ( $p=0.058, \mathrm{HR}, 4.72[95 \% \mathrm{Cl}, 0.61-36.57])$. Similarly, T790M-negative patients significantly showed shorter PFS than patients with T790M mutation ( $p=0.010$, median survival, 5.0 months, ratio, $0.5[95 \% \mathrm{Cl}, 0.23-1.08]$ vs 10.0 months, ratio, $2.5[95 \% \mathrm{Cl}, 0.92-4.33] ; \mathrm{HR}, 2.37[95 \% \mathrm{Cl}, 0.92-6.09]$ ), while OS was not statistically significant (Table 4). There was significant difference in the response rate between the T790M-positive and T790M-negative patients $(57.14 \%$ vs $7.69 \%$, respectively; $p=0.004$, Chisquared test). Furthermore, radio/chemotherapy (others) was significantly associated with poor PFS and OS in the multivariate analysis $(p=0.073$ and $p=$ 0.049; age, sex, history of smoking, type of treatment, EGFR status, EGFR T790M mutation, and concomitant mutations were entered into the multivariable Cox proportional hazards regression model) (Table 4). Interestingly, concomitant genetic alterations were significantly associated with a poor PFS for patients receiving third-generation EGFR-TKIs with T790M mutation as shown in Fig. 5 ( $p=0.0374$, median survival, 13.0 months, ratio,1.37 [95\% Cl, $0.39-4.76]$ vs 9.5 months, ratio, 0.73[95\% Cl, 0.21-2.54]; HR, 0.33 [95\% Cl, 0.12-0.87]). 
Table 4

Treatment outcomes for patients with EGFR-mutant advanced non-small cell lung cancer treated with EGFR TKIs.

\begin{tabular}{|c|c|c|c|c|c|c|c|c|c|c|}
\hline \multirow[t]{3}{*}{ Variable } & & \multirow[t]{3}{*}{ NO. (\%) } & \multicolumn{2}{|c|}{ Objective Response } & \multicolumn{4}{|c|}{ Progression-Free Survival } & \multicolumn{2}{|c|}{ Overall Survival } \\
\hline & & & \multirow[b]{2}{*}{ NO. (\%) } & \multirow[b]{2}{*}{$\begin{array}{l}\mathrm{P} \\
\text { Value }\end{array}$} & \multicolumn{2}{|l|}{ Univariate } & \multicolumn{2}{|l|}{ Multivariable* } & \multicolumn{2}{|l|}{ Univariate } \\
\hline & & & & & $\mathrm{HR}(95 \% \mathrm{Cl})$ & $\begin{array}{l}\mathrm{P} \\
\text { Value }\end{array}$ & $\mathrm{HR}(95 \% \mathrm{Cl})$ & $\begin{array}{l}\mathrm{P} \\
\text { Value }\end{array}$ & $\mathrm{HR}(95 \% \mathrm{Cl})$ & $\begin{array}{l}\mathrm{P} \\
\text { Value }\end{array}$ \\
\hline \multicolumn{11}{|c|}{$\begin{array}{l}\text { Post-TKI } \\
\text { treatments }\end{array}$} \\
\hline & $\begin{array}{l}\text { Third-generation } \\
\text { EGFR-TKIs }\end{array}$ & $24(70.59 \%)$ & $13(54.17 \%)$ & \multirow[t]{2}{*}{0.003} & 1(Reference) & \multirow[t]{2}{*}{ <. 0001} & 1(Reference) & \multirow[t]{2}{*}{0.073} & 1(Reference) & \multirow[t]{2}{*}{0.058} \\
\hline & Radio/Chemotherapy & $10(29.41 \%)$ & $0(0.0 \%)$ & & $\begin{array}{l}4.49(1.36- \\
14.76)\end{array}$ & & $\begin{array}{l}2.98(0.91- \\
9.82)\end{array}$ & & $\begin{array}{l}4.72(0.61- \\
36.57)\end{array}$ & \\
\hline \multicolumn{11}{|l|}{$\begin{array}{l}\text { EGFR } \\
\text { status }^{\#}\end{array}$} \\
\hline & Exon 19 deletion & $23(67.65 \%)$ & $9(39.13 \%)$ & \multirow[t]{2}{*}{0.877} & 1(Reference) & \multirow[t]{2}{*}{0.272} & 1(Reference) & \multirow[t]{2}{*}{0.727} & 1(Reference) & \multirow[t]{2}{*}{0.874} \\
\hline & Exon 21 mutation & $11(32.35 \%)$ & $4(36.36 \%)$ & & $\begin{array}{l}1.46(0.66- \\
3.23)\end{array}$ & & $\begin{array}{l}0.85(0.34- \\
2.13)\end{array}$ & & $\begin{array}{l}1.15(0.19- \\
7.12)\end{array}$ & \\
\hline \multicolumn{11}{|c|}{$\begin{array}{l}\text { EGFR } \\
\text { T790M } \\
\text { mutation\# }\end{array}$} \\
\hline & Positive & $21(61.76 \%)$ & $12(57.14 \%)$ & \multirow[t]{2}{*}{0.004} & 1(Reference) & \multirow[t]{2}{*}{0.010} & 1(Reference) & \multirow[t]{2}{*}{0.017} & 1(Reference) & \multirow[t]{2}{*}{0.584} \\
\hline & Negative & 13(38.24\%) & $1(7.69 \%)$ & & $\begin{array}{l}2.37(0.92- \\
6.09)\end{array}$ & & $\begin{array}{l}4.92(1.33- \\
18.16)\end{array}$ & & $\begin{array}{l}0.44(0.07- \\
2.72)\end{array}$ & \\
\hline \multicolumn{11}{|c|}{$\begin{array}{l}\text { Concomitant } \\
\text { mutation" }\end{array}$} \\
\hline & Yes & $24(70.59 \%)$ & $9(37.5 \%)$ & \multirow[t]{2}{*}{0.891} & 1(Reference) & \multirow[t]{2}{*}{0.479} & 1(Reference) & \multirow[t]{2}{*}{0.245} & 1(Reference) & \multirow[t]{2}{*}{0.553} \\
\hline & No & $10(29.41 \%)$ & $4(40 \%)$ & & $\begin{array}{l}0.78(0.37- \\
1.64)\end{array}$ & & $\begin{array}{l}0.58(0.23- \\
1.46)\end{array}$ & & $\begin{array}{l}0.53(0.08- \\
3.44)\end{array}$ & \\
\hline \multicolumn{11}{|c|}{ Abbreviations: EGFR, epidermal growth factor receptor; TKI, tyrosine kinase inhibitor } \\
\hline \multicolumn{11}{|c|}{$\begin{array}{l}\text { *Age (stratified by } 65 \text { years old), sex, history of smoking, type of TKI, EGFR status, EGFR T790M mutation, and concomitant mutations were entered into the } \\
\text { proportional hazards regression model. }\end{array}$} \\
\hline
\end{tabular}

\section{Resistance mechanisms to third-generation EGFR-TKI treatment}

After third-generation EGFR-TKI treatment, 22 patients presented PD and two patients presented SD. The analysis of putative mechanisms of resistance in these patients showed that the activation of known by-pass signaling pathways was observed in seven EGFR T790M-positive patients with PD, including three with PIK3CA mutation, two with MET amplification, one with PTEN deletion and one with STK11 mutation, excepting one with KRAS activating mutation in EGFR T790M-negative patients. Nine progressive patients presented other concomitant genetic alterations, including TP53, RB1, NOTCH1, FANCA, CTNNB1, and BRCA1. Notably, both patients without disease progression had co-mutations in the TP53. Moreover, in five patients (30\%) (\#1, \#3, \#6, \#7, \#40), no mechanisms of resistance to third-generation EGFR-TKIs were detected (Table 5). 
Table 5

Results of performed tissue re-biopsies after progression to third-generation EGFR-TKIs.

\begin{tabular}{|c|c|c|c|c|c|c|c|c|c|}
\hline \multicolumn{3}{|c|}{ T790M+ } & \multicolumn{7}{|c|}{ T790M- } \\
\hline patients & Mutations & Best Response & progression & PFS & patients & Mutations & Best Response & progression & PFS \\
\hline$\# 1$ & n.d & PR & yes & 17 & \#7 & n.d & SD & yes & 3 \\
\hline \#3 & n.d & PR & yes & 12 & \#22 & TP53, KRAS & SD & yes & 6 \\
\hline$\# 4$ & c-MET & PD & yes & 0 & \#38 & TP53 & SD & yes & 6 \\
\hline \#5 & PTEN & SD & yes & 3 & & & & & \\
\hline \#6 & n.d & SD & yes & 13 & & & & & \\
\hline$\# 10$ & TP53, STK11 & PR & yes & 9 & & & & & \\
\hline$\# 11$ & PIK3CA, NOTCH1 & SD & yes & 11 & & & & & \\
\hline$\# 12$ & TP53, RB1 & $\mathrm{PR}$ & yes & 14 & & & & & \\
\hline \#14 & BRCA1 & PR & yes & 10 & & & & & \\
\hline$\# 15$ & PIK3CA, TP53 & PR & yes & 5 & & & & & \\
\hline$\# 16$ & FANCA, CTNNB1 & SD & yes & 12 & & & & & \\
\hline \#18 & TP53 & SD & yes & 13 & & & & & \\
\hline$\# 20$ & TP53 & PR & no & 12 & & & & & \\
\hline$\# 24$ & PIK3CA, FANCA, CTNNB1 & PR & yes & 7 & & & & & \\
\hline \#26 & TP53 & PR & yes & 11 & & & & & \\
\hline \#28 & TP53 & SD & yes & 9 & & & & & \\
\hline \#30 & TP53 & PR & yes & 10 & & & & & \\
\hline \#32 & TP53, RB1 & SD & yes & 5 & & & & & \\
\hline \#34 & c-MET, TP53 & PR & yes & 6 & & & & & \\
\hline \#36 & TP53 & PR & no & 9 & & & & & \\
\hline \#40 & n.d & PR & yes & 7 & & & & & \\
\hline
\end{tabular}

\section{Discussion}

EGFR mutation has long been considered as the most important prognostic factor in patients with advanced NSCLC in the EGFR-TKI era. And EGFR-TKIs were more effective in treating advanced NSCLC than chemotherapy. However, as resistance develops, re-biopsy will play a significant role in guiding the use of third-generation EGFR-TKIs. Also, it provides a better understanding of the underlying resistance mechanisms for third-generation EGFR-TKIs to optimize clinical outcomes. Therefore, we prospectively enrolled 39 patients to study the status of re-biopsy and retrospectively analyzed 34 EGFR-mutant patients, who were diagnosed with advanced NSCLC after failure to first-line EGFR-TKI treatment.

A total of 34 pairs of tissue-to-blood paired specimens were successfully biopsied, and histopathological typing and gene detection of tissue and blood were performed with a success rate of $91.89 \%$ (34/37). The success rate reported in previous biopsy studies ranged from $73-95 \%$, and our results are similar to previous studies [13-16]. The reason for our higher success rate may be that we performed more than three biopsy times per lesion. For the three cases that failed to obtain enough tumor tissues for gene detection, the puncture sites were all primary lung lesions, and the pathological characteristics suggested that blood clots and some scattered lung tissues were presented under the microscope, but no tumor cells were observed. This may be due to the fact that the lesion contains more fibrous tissue components or necrosis and blood clots after target treatment, which may increase the difficulty in the process of rebiopsy. Even if some heterotypic cells may exist in the on-site cytology, the number and proportion of tumor cells cannot be guaranteed. This also indicates the difficulty of re-biopsy after the first-generation EFGR-TKI resistance. Another reason for the lack of tumor cells in the re-biopsy samples may be due to the heterogeneity of the tumor tissue. Previous studies have shown that larger differences can occur between primary tumors and their metastases or tumors of different pathological subtypes of the same tissue [17]. Xie et al. proved that there was no significant difference in PFS between patients with partially matched mutational profiles between primary tumors and metastatic lymph nodes and those having $100 \%$ concordance rate. It indicated that the genetic profiles of both primary lesions and metastatic lymph nodes could be a guidance of NSCLC targeted therapy. As well as other studies, it was confirmed that the genetic mutations in the primary lung and metastases were similar in patients with advanced lung cancer [18-20]. Unfortunately, we were unable to confirm another re-biopsy in these three patients, so we were not sure whether we could obtain adequate, effective and qualified tissue specimens for histopathological typing and genetic testing. However, it may be considered that if the primary lung lesion biopsy fails to obtain a qualified specimen, other metastatic lesions and metastatic lymph nodes may be considered for genetic testing to guide follow-up treatment. 
In this study, we detected and identified potentially actionable alterations using both blood-derived ctDNA and tumor tissue by different platforms, especially for EGFR T790M mutation. Compared with traditional detection methods, NGS technology has better detection efficiency and higher throughput through tissue and blood samples. It can detect mutations, insertions, rearrangements and copy number variations quantitatively at the same time, and also significantly save cost and time in large-scale sequencing. Concordance rates between tissue and ctDNA NGS appeared high here, although there were differences in genomic alterations detected. However, compared with the efficacy of genetic testing for tissue biopsy specimens, the efficacy of blood test for T790M still has more false negatives, resulting in lower sensitivity. Meanwhile, there was also one patient with T790M mutation in plasma but negative in tissue. We combined the efficacy with the test results to consider that the tissue test results were also false negative, suggesting that tissue and ctDNA assay provided complementary results. Therefore, domestic and foreign guidelines recommend that when the patient's physical condition is tolerable and tissue specimens are available, tissue biopsy should be preferred as the first choice for re-biopsy after EGFR-TKI resistance. When tissue specimens are not available or tissue specimens are unqualified and patients refuse to perform, in the case of a re-biopsy, liquid biopsy techniques can be a powerful complement.

For genetic alterations and resistance mechanisms, we retrospectively analyzed 34 patients with mutations detected by both ctDNA and tissue NGS. Among them, $88.23 \%$ (30/34) of patients harbored concomitant mutations detected by at least one NGS assay. And co-occurring genetic alterations were negatively correlated with the response to the treatment, which may be plausibly due to the bypass activation of survival signaling pathways or tumor heterogeneity. Consistent with recent studies [12], patients with 19del survived longer than patients with 21L858R mutation. Of interest, a higher incidence of concomitant mutations in patients was also detected with 21L858R, although there was no significant difference which was not shown in the results. As we know, the presence of EGFR T790M mutation in resistant patients after first-line EGFR-TKI therapy was significantly associated with better efficacy of third-generation EGFR-TKIs, which provides a rationale for the superiority of third-generation EGFR-TKI therapy over other therapies. In multivariable analysis, the EGFR T790M mutation was still significantly associated with survival.

To study the potential mechanisms of resistance, re-biopsy at progression to third-generation EGFR-TKIs was performed in 24 patients. Activation of known by-pass signaling pathways as mechanism of resistance was represented, and one patient with STK11 mutation, one with PTEN deletion, one with KRAS mutation, two with MET amplification and three with PIK3CA mutation were observed. STK11 mutation presented accompanying with TP53 mutation in an EGFR T790M-positive patient, which had been reported as a mediator of the cold tumor immune microenvironment and a major driver of primary resistance to PD-1 axis inhibitors in non-squamous lung adenocarcinoma [21]. PTEN loss was previously described as a mechanism of resistance to first-generation EGFRTKIs [22]. While Kim et al [23]. reported a following increase of the proportion of tumors with PTEN deletions in post-treatment tumors and the gradual increase of PTEN deletions might contribute to focal progression to Osimertinib. MET amplification was already mentioned as a possible mechanism of resistance to Osimertinib, which has been considered as the very common findings of acquired resistance under first-generation EGFR-TKIs [24-26], and described in the literature at frequencies ranging from 5-50\% [27, 28]. Particularly, activating mutations of the catalytic subunit alpha (PIK3CA) of PI3K lipid kinases family was associated with poor PFS in our cohort including a case of small cell lung cancer (SCLC) transformation, since activation of $\mathrm{PI} 3 \mathrm{~K} / \mathrm{AKT} / \mathrm{mTOR}$ signaling pathway was not mutually exclusive with other carcinogenic driving mechanisms. The shorter median survival time in patients with co-existing PIK3CA and EGFR mutations suggested a possible synergistic effect due to stronger activation of relevant downstream signals [29, 30]. The results suggested a potential role of PIK3CA-inhibitor, alone or in combination, to accurately overcome this resistance.

The main limitations of this study were sample size, single center design and lack of continuous biopsy. Furthermore, data from our NGS hotspots (excluding other mutations, copy number changes, or chromosomal abnormalities) may represent an underestimation of concomitant mutations and impede further analysis of signaling pathways or cloning.

\section{Conclusions}

In conclusion, using multi-platforms to perform detection of EGFR T790M mutation on EGFR-TKI resistant re-biopsy tissue and blood samples is feasible, and the consistency between tissue and blood samples is high. They can provide complementary results mutually. Our study also highlights the importance of rebiopsy and molecular diagnosis during disease progression in patients treated by EGFR-TKIs for oncogene-addicted NSCLC. And concomitant genetic alterations may affect response to treatment and decision of sequential therapy strategy.

\section{Abbreviations}

\section{ARMS-PCR}

amplification refractory mutation system-polymerase chain reaction;

$\mathrm{CR}$

complete response;

ctDNA

circulating tumor DNA;

ddPCR

digital droplet PCR;

ECOG

Eastern Cooperative Oncology Group;

EGFR

epidermal growth factor receptor;

EGFR-TKI

epidermal growth factor receptor-tyrosine kinase inhibitor; NGS 
next-generation sequencing;

NSCLC

non-small cell lung cancer;

ORR

objective response rate;

os

overall survival;

PD

progressive disease;

PFS

progression-free survival;

PR

partial response;

PS

performance status;

RECIST

Response Evaluation Criteria in Solid Tumors;

ROSE

rapid on-site evaluation;

SD

stable disease;

TBB

transbronchial biopsy;

TBLB

transbronchial lung biopsy;

TBNA

transbronchial needle aspiration;

TTNA

transthoracic needle aspiration.

\section{Declarations}

\section{Ethics approval and consent to participate}

The study was approved by the Ethics Committee of Shanghai Chest Hospital, and the ethical approval number was KS1703. All patients were fully informed and signed informed consent. The clinical trial registration was carried out on ClinicalTrials.gov. (NCT03309462).

\section{Consent for publication}

Not applicable.

\section{Availability of data and materials}

The datasets used and/or analyzed during the current study are available from the corresponding author on reasonable request.

\section{Competing Interests}

The authors declare that they have no competing interests.

\section{Funding}

This work was supported by National Key R\&D Program of China (2017YFC0112700); Shanghai Municipal Health and Medical Talents Training Program (2018BR09); Shanghai Municipal Education Commission-Gaofeng Clinical Medicine Grant Support (20181815).

\section{Authors' Contributions}

J. S. and L. X. contributed to the study design, subjects enrolled, acquisition of specimens, data analysis and revising the manuscript. Y. Z. contributed to the data collection, analysis and interpretation, drafting and revising the manuscript. F. X. contributed to analysis and revising the manuscript. X. Z. and Y. L. contributed to the acquisition of specimens and reviewing the manuscript. L. Z. contributed to the pathological analysis and reviewing the manuscript. All authors read and approved the final manuscript. 


\section{Acknowledgements}

We would like to express our gratitude to Dr. Xueyan Zhang, Dr. Huimin Wang and Dr. Yuqing Lou, who have offered us valuable resource and suggestions during this study. We thank Shanghai Tongshu Biotechnology Co., Ltd. for technical support.

\section{References}

1. Bray F, Ferlay J, Soerjomataram I, Siegel RL, Torre LA, Jemal A. Global cancer statistics 2018: GLOBOCAN estimates of incidence and mortality worldwide for 36 cancers in 185 countries. CA Cancer J Clin. 2018;68:394-424. https://doi.org/10.3322/caac.21492

2. Govindan R, Page N, Morgensztern D, et al. Changing epidemiology of small-cell lung cancer in the United States over the last 30 years: analysis of the surveillance, epidemiologic, and end results database. J Clin Oncol. 2006;24:4539-4544. https://doi.org/10.1200/JC0.2005.04.4859

3. Lindeman NI, Cagle PT, Beasley MB, et al. Molecular testing guideline for selection of lung cancer patients for EGFR and ALK tyrosine kinase inhibitors: guideline from the College of American Pathologists, International Association for the Study of Lung Cancer, and Association for Molecular Pathology. J Thorac Oncol. 2013;8:823-859. https://doi.org/10.1097/JT0.0b013e318290868f

4. Fang S, Wang Z. EGFR mutations as a prognostic and predictive marker in non-small-cell lung cancer. Drug Des Devel Ther. 2014;8:1595-1611. https://doi.org/10.2147/DDDT.S69690

5. Jackman D, Pao W, Riely GJ, et al. Clinical definition of acquired resistance to epidermal growth factor receptor tyrosine kinase inhibitors in non-small-cell lung cancer. J Clin Oncol. 2010;28:357-360. https://doi.org/10.1200/JC0.2009.24.7049

6. Cross DAE, Ashton SE, Ghiorghiu S, et al. AZD9291, an irreversible EGFR TKI, overcomes T790M-mediated resistance to EGFR inhibitors in lung cancer. Cancer Discov. 2014;4:1046-1061. https://doi.org/10.1158/2159-8290.CD-14-0337

7. Attili I, Karachaliou N, Conte P, Bonanno L, Rosell R. Therapeutic approaches for T790M mutation positive non-small-cell lung cancer. Expert Rev Anticancer Ther. 2018;18:1021-1030. https://doi.org/10.1080/14737140.2018.1508347

8. National Comprehensive Cancer Network. NCCN clinical practice guidelines in oncology. Non-small cell lung cancer. https://www.nccn.org/professionals/physician_gls/default.aspx\#nscl; 2020 Accessed 29 February, 2020.

9. Tetsu O, Hangauer MJ, Phuchareon J, Eisele DW, McCormick F. Drug Resistance to EGFR Inhibitors in Lung Cancer. Chemotherapy. 2016;61:223-235. https://doi.org/10.1159/000443368

10. Hata A, Katakami N, Yoshioka H, et al. Spatiotemporal T790M Heterogeneity in Individual Patients with EGFR-Mutant Non-Small-Cell Lung Cancer after Acquired Resistance to EGFR-TKI. J Thorac Oncol. 2015;10:1553-1559. https://doi.org/10.1097/jto.0000000000000647

11. Blakely CM, Watkins TBK, Wu W, et al. Evolution and clinical impact of co-occurring genetic alterations in advanced-stage EGFR-mutant lung cancers. Nat Genet. 2017;49:1693-1704. https://doi.org/10.1038/ng.3990

12. Hong S, Gao F, Fu S, et al. Concomitant Genetic Alterations With Response to Treatment and Epidermal Growth Factor Receptor Tyrosine Kinase Inhibitors in Patients With EGFR-Mutant Advanced Non-Small Cell Lung Cancer. JAMA Oncol. 2018;4:739-742. https://doi.org/10.1001/jamaoncol.2018.0049

13. Nosaki K, Satouchi M, Kurata T, et al. Re-biopsy status among non-small cell lung cancer patients in Japan: A retrospective study. Lung Cancer. 2016;101:1-8. https://doi.org/10.1016/j.lungcan.2016.07.007

14. Kirita K, Izumo T, Matsumoto Y, Hiraishi Y, Tsuchida T. Bronchoscopic Re-biopsy for Mutational Analysis of Non-small Cell Lung Cancer. Lung. 2016;194:371-378. https://doi.org/10.1007/s00408-016-9864-5

15. Chouaid C, Dujon C, Do P, et al. Feasibility and clinical impact of re-biopsy in advanced non small-cell lung cancer: a prospective multicenter study in a real-world setting (GFPC study 12-01). Lung Cancer. 2014;86:170-173. https://doi.org/10.1016/j.lungcan.2014.08.016

16. Bosc C, Ferretti GR, Cadranel J, et al. Rebiopsy during disease progression in patients treated by TKI for oncogene-addicted NSCLC. Target Oncol. 2015;10:247-253. https://doi.org/10.1007/s11523-014-0332-y

17. McGranahan N, Swanton C. Clonal Heterogeneity and Tumor Evolution: Past, Present, and the Future. Cell. 2017;168:613-628. https://doi.org/10.1016/j.cell.2017.01.018

18. Xie F, Zhang Y, Mao X, et al. Comparison of genetic profiles among primary lung tumor, metastatic lymph nodes and circulating tumor DNA in treatmentnaive advanced non-squamous non-small cell lung cancer patients. Lung Cancer. 2018;121:54-60. https://doi.org/10.1016/j.lungcan.2018.05.002

19. Sherwood J, Dearden S, Ratcliffe M, Walker J. Mutation status concordance between primary lesions and metastatic sites of advanced non-small-cell lung cancer and the impact of mutation testing methodologies: a literature review. J Exp Clin Cancer Res. 2015;34:92. https://doi.org/10.1186/s13046015-0207-9

20. Wang S, Wang Z. Meta-analysis of epidermal growth factor receptor and KRAS gene status between primary and corresponding metastatic tumours of non-small cell lung cancer. Clin Oncol (R Coll Radiol). 2015;27:30-39. https://doi.org/10.1016/j.clon.2014.09.014

21. Skoulidis F, Goldberg ME, Greenawalt DM, et al. STK11/LKB1 Mutations and PD-1 Inhibitor Resistance in KRAS-Mutant Lung Adenocarcinoma. Cancer Discov. 2018;8:822-835. https://doi.org/10.1158/2159-8290.CD-18-0099

22. Sos ML, Koker M, Weir BA, et al. PTEN loss contributes to erlotinib resistance in EGFR-mutant lung cancer by activation of Akt and EGFR. Cancer Res. 2009;69:3256-3261. https://doi.org/10.1158/0008-5472.CAN-08-4055

23. Kim TM, Song A, Kim DW, et al. Mechanisms of Acquired Resistance to AZD9291: A Mutation-Selective, Irreversible EGFR Inhibitor. J Thorac Oncol. 2015;10:1736-1744. https://doi.org/10.1097/JT0.0000000000000688

24. Sequist LV, Waltman BA, Dias-Santagata D, et al. Genotypic and histological evolution of lung cancers acquiring resistance to EGFR inhibitors. Sci Transl Med. 2011;3:75ra26. https://doi.org/10.1126/scitranslmed.3002003

Page $12 / 16$ 
25. Yu HA, Arcila ME, Rekhtman N, et al. Analysis of tumor specimens at the time of acquired resistance to EGFR-TKI therapy in 155 patients with EGFRmutant lung cancers. Clin Cancer Res. 2013;19:2240-2247. https://doi.org/10.1158/1078-0432.ccr-12-2246

26. Arcila ME, Oxnard GR, Nafa K, et al. Rebiopsy of lung cancer patients with acquired resistance to EGFR inhibitors and enhanced detection of the T790M mutation using a locked nucleic acid-based assay. Clin Cancer Res. 2011;17:1169-1180. https://doi.org/10.1158/1078-0432.ccr-10-2277

27. Oxnard GR, Hu Y, Mileham KF, et al. Assessment of Resistance Mechanisms and Clinical Implications in Patients With EGFR T790M-Positive Lung Cancer and Acquired Resistance to Osimertinib. JAMA Oncol. 2018;4:1527-1534. https://doi.org/10.1001/jamaoncol.2018.2969

28. Lin CC, Shih JY, Yu CJ, et al. Outcomes in patients with non-small-cell lung cancer and acquired Thr790Met mutation treated with osimertinib: a genomic study. Lancet Respir Med. 2018;6:107-116. https://doi.org/10.1016/S2213-2600(17)30480-0

29. Chaft JE, Arcila ME, Paik PK, et al. Coexistence of PIK3CA and other oncogene mutations in lung adenocarcinoma-rationale for comprehensive mutation profiling. Mol Cancer Ther. 2012;11:485-491. https://doi.org/10.1158/1535-7163.MCT-11-0692

30. Ludovini V, Bianconi F, Pistola L, et al. Phosphoinositide-3-Kinase Catalytic Alpha and KRAS Mutations are Important Predictors of Resistance to Therapy with Epidermal Growth Factor Receptor Tyrosine Kinase Inhibitors in Patients with Advanced Non-small Cell Lung Cancer. J Thorac Oncol. 2011;6:707-

715. https://doi.org/10.1097/JT0.0b013e31820a3a6b

\section{Figures}

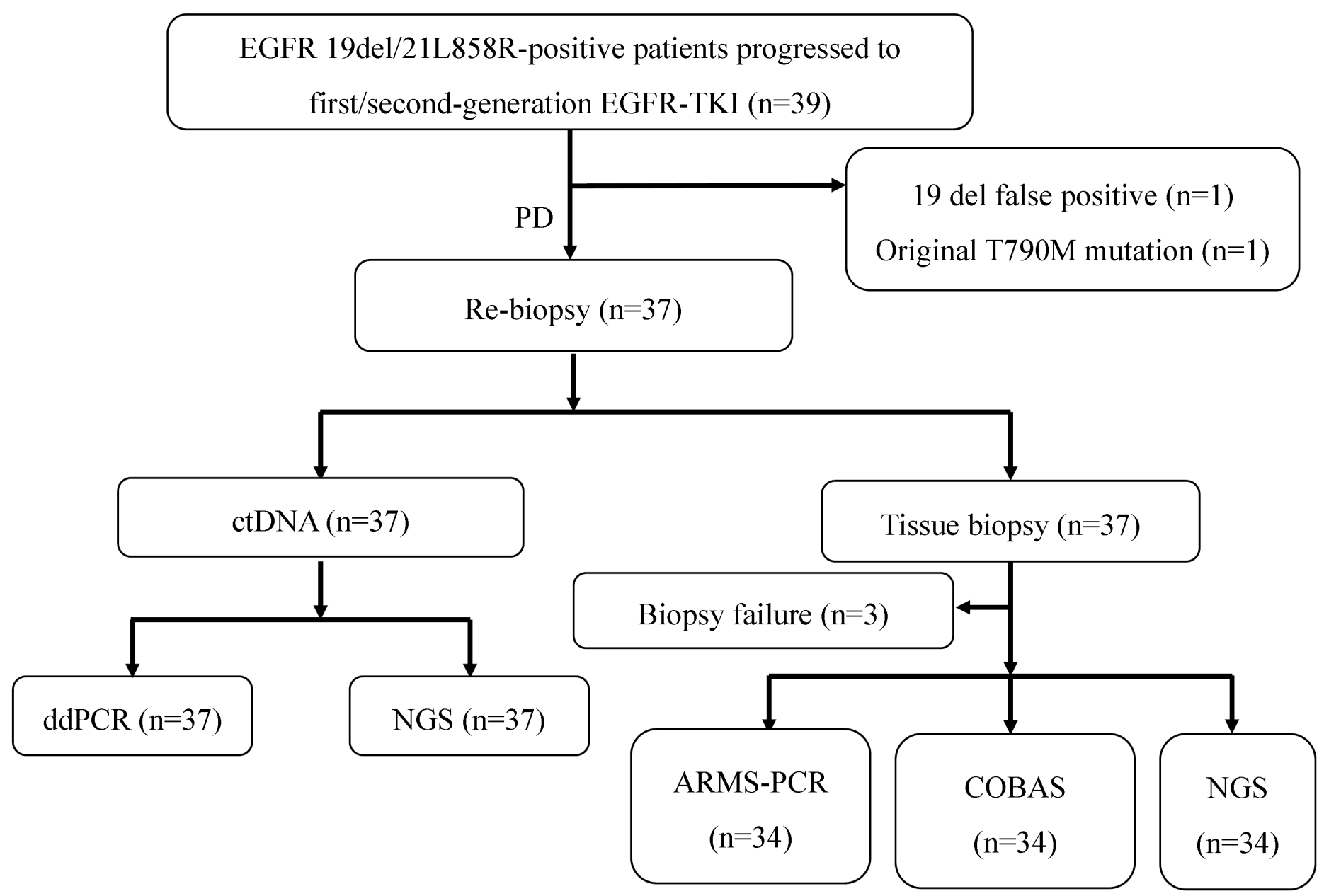

Figure 1

Flow chart of eligible population. A total of 39 patients with lung cancer diagnosed with EGFR 19del or EGFR 21L858R mutation positive were resistant to the first and second-generation EGFR-TKIs after treatment and enrolled. Among them, when NGS re-examined the genes in the first biopsy sample, 1 case was excluded due to the result indicating that EGFR 19del was false positive, and 1 case was excluded due to the result indicating mutation containing EGFR 20T790M. Thirty-seven patients had tissue gene testing and blood gene testing. ARMS-PCR, amplification refractory mutation system polymerase chain reaction; ctDNA, circulating tumor DNA; ddPCR, digtal droplet polymerase chain reaction; EGFR, epidermal growth factor receptor; EGFR-TKI, epidermal growth factor receptor- tyrosine kinase inhibitor; NGS, next-generation sequencing; $P D$, progressive disease 
A
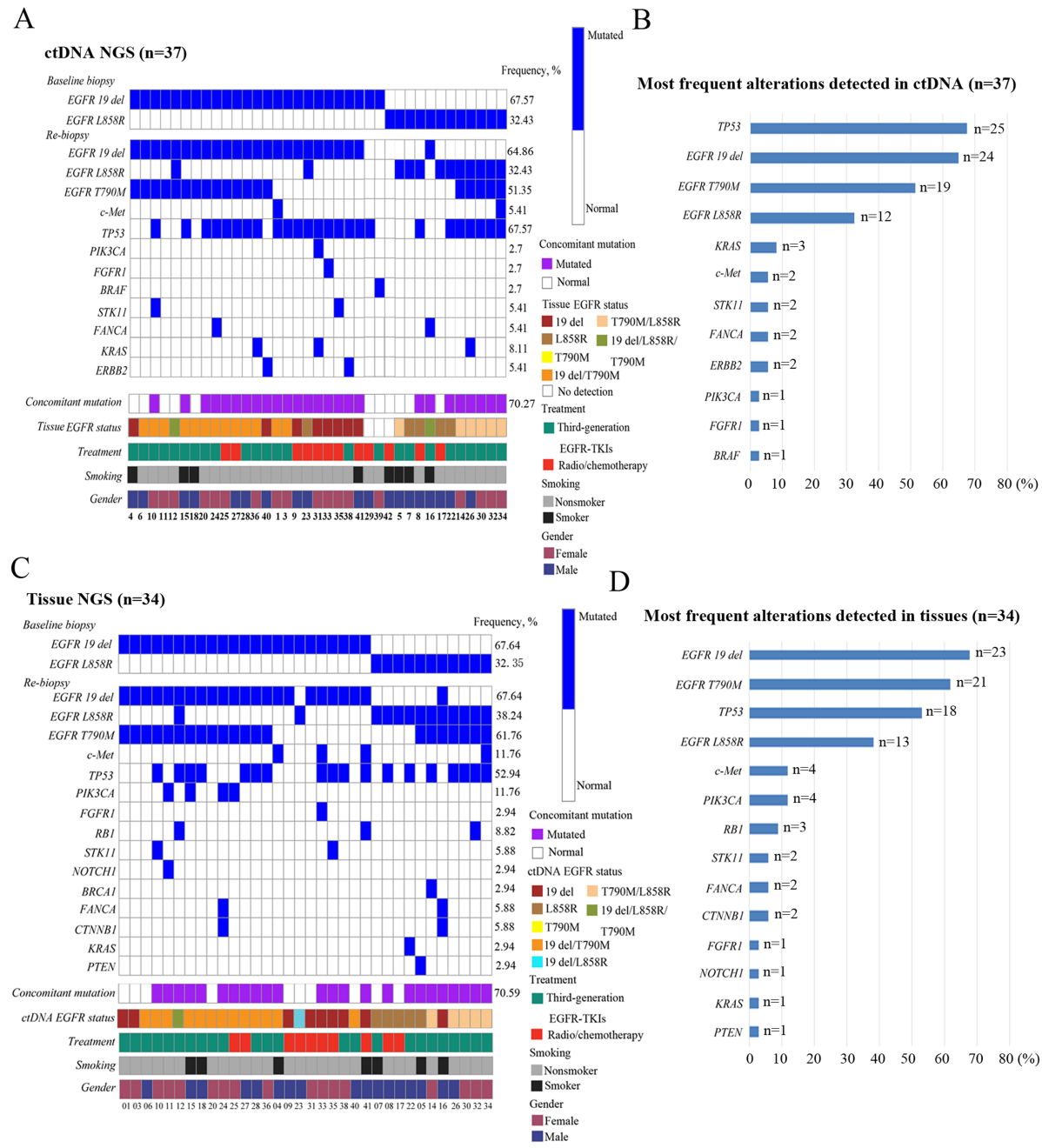

Figure 2

Genetic alterations of enrolled patients by ctDNA and tissue NGS. (A) Oncoprint of ctDNA NGS alterations ( $n=37$ ). Synonymous alterations and variants of unknown significance were excluded. All 37 patients were tested for ctDNA, but only 34 of them were also tested for tissue NGS. Each vertical bar represents a patient. (B) Most frequent alterations identified by plasma-derived ctDNA NGS ( $n=37)$. (C) Oncoprint of tissue NGS ( $n=34)$. (D) Most frequent alterations identified by tissue NGS ( $n=34)$. ctDNA, circulating tumor DNA; EGFR, epidermal growth factor receptor; EGFR-TKI, epidermal growth factor receptor- tyrosine kinase inhibitor; NGS, next-generation sequencing

A

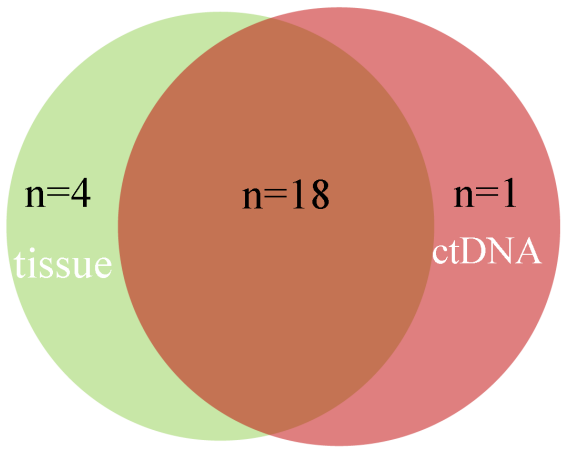

B

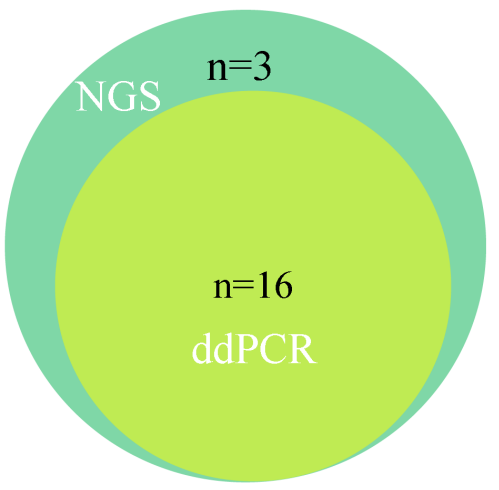

C

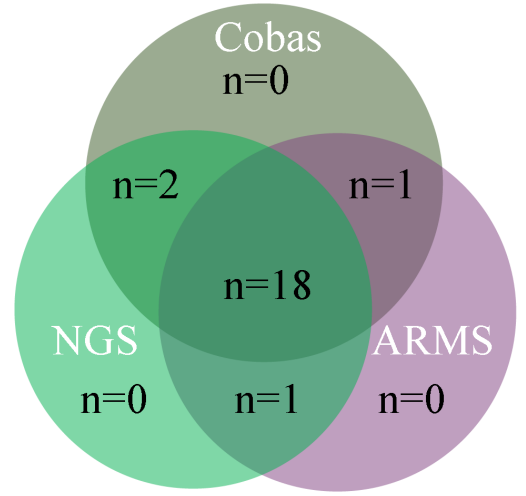

Tissue VS ctDNA ctDNA

Tissue

Figure 3 
The detection efficiency of different detection platforms for EGFR 20T790M mutation. (A) T790M mutation was detected and compared by both ctDNA and tissue assays and as illustrated by the Venn diagrams. (B) T790M mutation was detected and compared by NGS and ddPCR in plasma samples. (C) T790M mutation was detected and compared by ARMS-PCR, Cobas ${ }^{\circledR}$ ARMS-PCR and NGS in tissue samples. ARMS-PCR, amplification refractory mutation system polymerase chain reaction; ctDNA, circulating tumor DNA; ddPCR, digtal droplet polymerase chain reaction; EGFR, epidermal growth factor receptor; NGS, nextgeneration sequencing

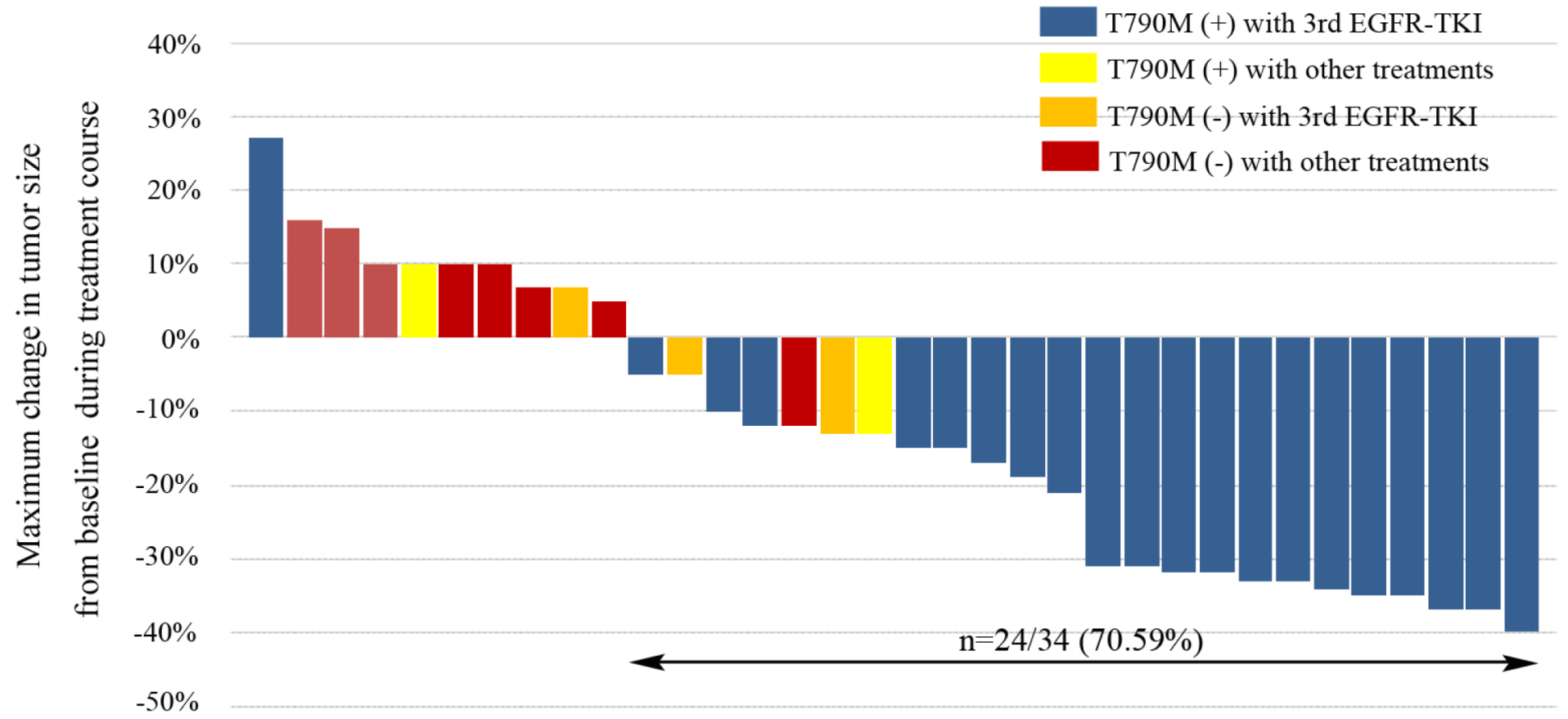

04330908273135172223183806284107251116320305152010363040241234012614

\section{Figure 4}

Maximum change in tumor size from baseline in individual patients over the course of treatment. Changes in tumor size (diameter) were assessed in patients with or without T790M mutation treated with the third-generation EGFR-TKIs or other treatments. Tumor shrinkage relative to baseline was observed in 70.59 $\%$ of patients. EGFR-TKI, epidermal growth factor receptor- tyrosine kinase inhibitor 
A

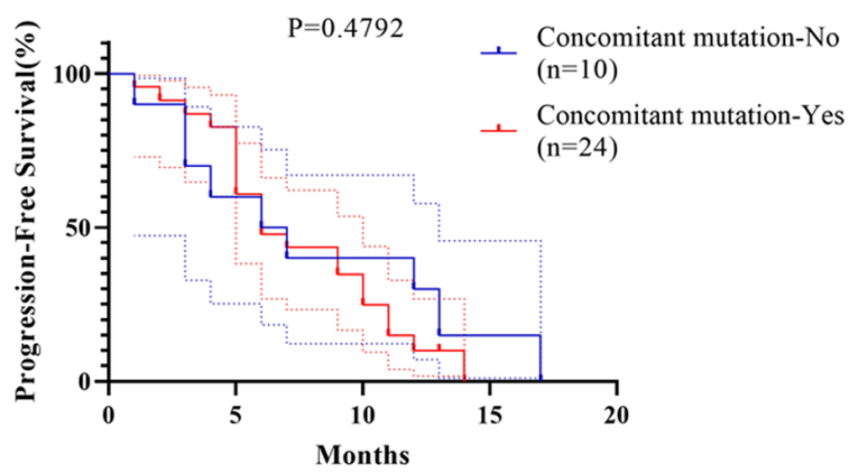

B

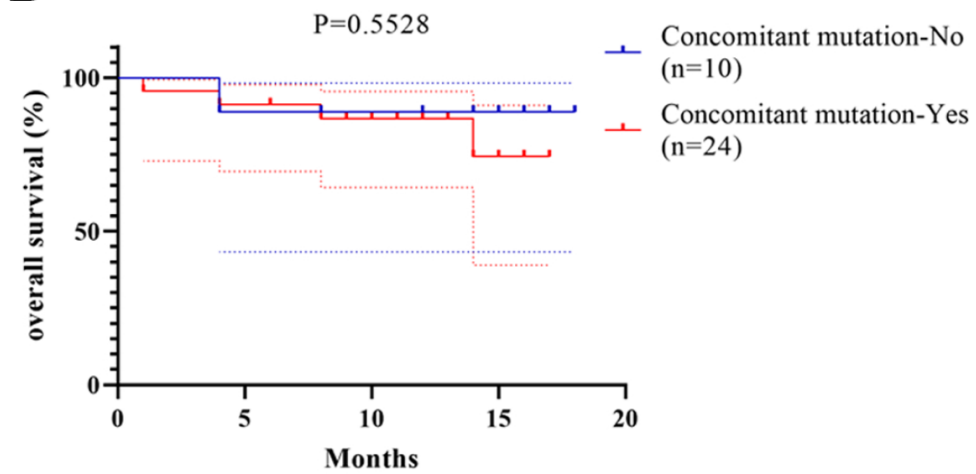

EGFR T790M mutations + Third-generation EGFR-TKIs treatment
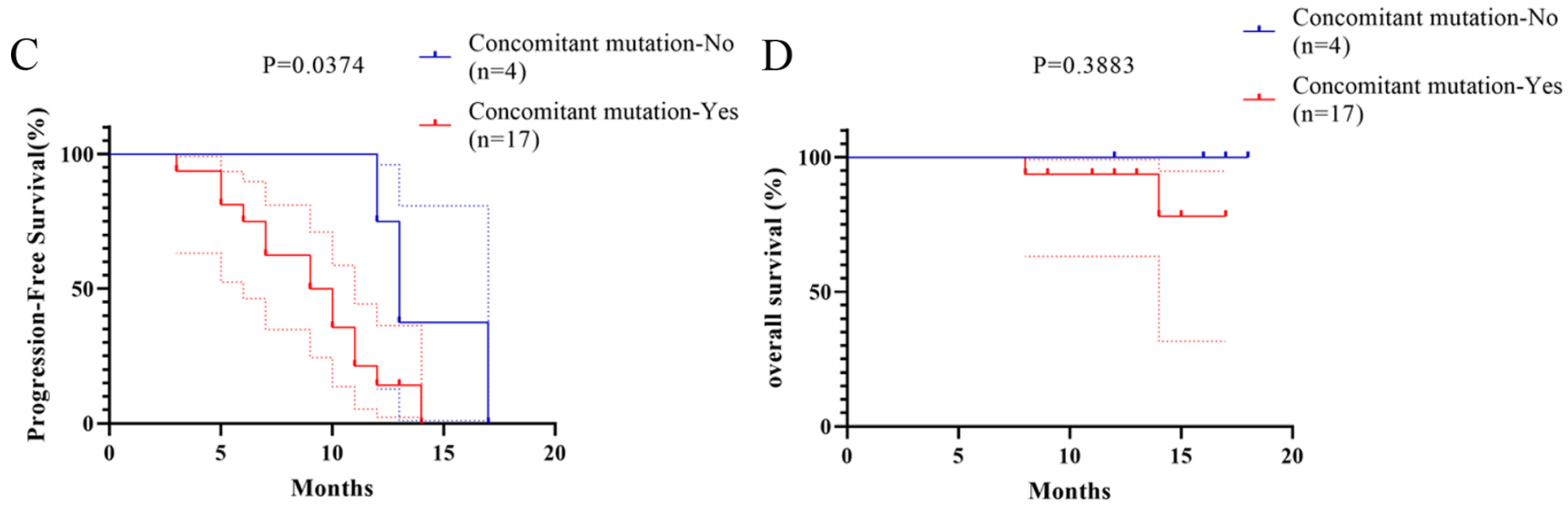

\section{Figure 5}

Survivals of patients included in retrospective analysis according to tissue re-biopsy molecular status and treatments. (A) Kaplan-Meier curves of PFS in patients whose tissue re-biopsy had concomitant mutations compared with those without concomitant mutations. (B) Kaplan-Meier curves of OS in patients whose tissue re-biopsy had concomitant mutations compared with those without concomitant mutations. (C) Kaplan-Meier curves of PFS in patients with EGFR T790M mutation whose tissue re-biopsy had concomitant mutations compared with those without concomitant mutations after receiving thirdgeneration EGFR-TKIs treatment. (D) Kaplan-Meier curves of OS in patients with EGFR T790M mutation whose tissue re-biopsy had concomitant mutations compared with those without concomitant mutations after receiving third-generation EGFR-TKIs treatment. EGFR, epidermal growth factor receptor; EGFR-TKI, epidermal growth factor receptor- tyrosine kinase inhibitor; OS, overall survival; PFS, progression-free survival 